\title{
Valorisation des sciures du bois de Moringa oleifera dans le traitement physico-chimique des lixiviats du lieu d'enfouissement sanitaire (LES) de Ouèssè/Ouidah (Sud Bénin)
}

\author{
"Roger Gérard JOSSE1, Rock Modéran TOKLO1', Pierre DOSSOU-YOVO2, Jacques K. FATOMBI ${ }^{3}$ \\ 1. Laboratoire d'Analyse Physico-chimique des Milieux Aquatiques (LAPMIA/FAST/CHIMIE/UAC) BP 526 Cotonou \\ (Rép. Bénin) \\ 2. Laboratoire de Recherche en Traitement et Conservation des Produits Halieutiques (FAST/ CHIMIE /UAC) BP \\ 526 Cotonou (Rép. Bénin) \\ 3. Laboratoire d'Expertise et de Recherche en Chimie de l'Eau et de l'Environnement (LERCEE / FAST/ CHIMIE/ \\ UAC) BP 526 Cotonou (Rép. BENIN) \\ * Auteur correspondant : jossserogergerard@yahoo.fr
}

Original submitted in on $13^{\text {th }}$ November 2015. Published online at www.m.elewa.org on $31^{\text {st }}$ December 2015 http://dx.doi.org/10.4314/jab.v96i1.8

\section{RÉSUMÉ}

Objectif: La présente étude vise à valoriser les biomatériaux (sciure brute; sciure activée et sciure calcinée) issus du bois de Moringa oleifera dans le traitement des lixiviats du lieu d'enfouissement sanitaire de Ouèssè/Ouidah (Sud Bénin).

Méthodologie et résultats: Plusieurs méthodes physico-chimiques d'analyse ont été utilisées au cours de ces travaux : oxymétrie, colorimétrie, spectrophotométrie, COT-métrie... Les résultats obtenus montrent que la sciure du bois calcinée permet de mieux traiter les lixiviats avec une diminution de la turbidité (95\%), de la matière organique $(90 \%)$, des solides totaux dissous $(88,53 \%)$, de la couleur $(69,74 \%)$ et de la matière en suspension $(61,70 \%)$; mais augmente légèrement la conductivité électrique. Ce coagulant influence le pH des lixiviats traités mais n'a pas d'effet sur la température.

Conclusion et Application : La sciure de bois calcinée permet d'obtenir une meilleure clarification des lixiviats. Ce travail devrait intéresser les pouvoirs politiques et particulièrement les autorités des villes de Cotonou et de Ouidah pour un meilleur traitement des lixiviats du lieu d'enfouissement sanitaire (LES).

Mots clés : LES, lixiviats, coagulant, sciure.

\begin{abstract}
Objective: This work aims to valorize biomaterials (raw sawdust; activated sawdust and calcined sawdust) stemming from Moringa oleifera's wood in the treatment of the leachates of the landfill area of Ouèssè / Ouidah (South of Benin).

Methodology and results : several physico-chemical methods of analysis were used during these works: oxymetry, colorimetry, spectrophotometry, COT-metry etc. The obtained results show that the sawdust of calcined wood allows to better treat the leachates with the reduction of the turbidity (95\%), of the organic matter $(90 \%)$, of the total dissolved substances $(88,53 \%)$, of the color $(69,74 \%)$ and of the suspended
\end{abstract}




\section{Josse et al. J. Appl. Biosci. 2015 Valorisation des sciures du bois de Moringa oleifera dans le traitement}

physico-chimique des lixiviats du lieu d'enfouissement sanitaire (LES) de Ouèssèl Ouidah (Sud Bénin)

materials $(61,70 \%)$; but increase slightly the electric conductivity. This coagulant influences the $\mathrm{pH}$ of the treated leachates but has no effect on the temperature.

Conclusion and Application: The calcined sawdust allows to obtain a better clarification of leachates. This work should interest the political powers and particularly the authorities of the cities of Cotonou and Ouidah for a better treatment of the leachates of LES.

Keywords: LES, leachates, coagulating, sawdust.

\section{INTRODUCTION}

Dans les pays en voie de développement, et en particulier au Bénin, le mode de gestion des déchets solides est la mise en décharge. Les eaux générées par les décharges sont appelées lixiviats et représentent un milieu complexe. La composition chimique des lixiviats dépend de la composition typologique des déchets stockés, des propriétés du sol, de la pluviométrie et de l'âge de la décharge (Baun et al, 2004). En effet, les lixiviats sont en générale riches en matières organique, inorganique et en métaux lourds (Toklo et al, 2015). Leur rejet à l'état brut, sans aucun traitement préalable, engendre des effets tels que la pollution des sols, la contamination des eaux souterraines et de surface. Aussi, les lixiviats peuvent causer de mauvaises odeurs et des aérosols, affectant ainsi la santé des individus. Afin de réduire le risque de pollution des eaux des milieux des décharges par les lixiviats,

\section{MATÉRIEL ET MÉTHODES}

Site de l'étude : Le lieu d'enfouissement sanitaire (LES) est situé le long de la route reliant la localité de Savi à la route nationale longeant le littoral et se trouve à environ $700 \mathrm{~m}$ au nord-ouest de l'intersection de la route de Savi et de la piste menant au village de Ouèssè (commune de Ouidah). Le site est situé à environ $5 \mathrm{~km}$ au nord de la route bitumée qui relie celle-ci à Savi (Toklo et al, 2015).

Points d'échantillonnage : Les prélèvements de lixiviat ont été réalisés à deux endroits du site de décharge. Les échantillons de lixiviat sont prélevés dans des flacons préalablement rincés à l'eau distillée puis stockés au laboratoire dans un réfrigérateur à la température de $4^{\circ} \mathrm{C}$.

Préparation des biomatériaux : Le biomatériau naturel utilisé est la sciure de bois provenant de la plante de Moringa oleifera (figure ${ }^{\circ} 1$ ). Les copeaux de bois ont été récoltés dans la ville d'Aplahoué en juillet 2015. Ils sont ensuite séchés au laboratoire à l'air libre pendant 30 jours puis broyés à l'aide d'un mortier de laboratoire ; la poudre résultante est tamisée. Dans un premier temps, nous plusieurs équipes de recherche (Holan et al, 1995 ; Ajmal et al, 1996 ; Chaterjee et al, 1996; Ajmal et al, 1998 ; Fatombi et al, 2007 ; Zalaghi et al, 2014) ont utilisé les sciures des bois tels que Abies magnifica, Mangifera indica, Tilia americana, Picea engelmani, Pinus roxburghi, Cedrus deodara, Pinus destflora, Fagas crenata, Bassial atifulia, Arundo donax, Juniperus phoenice et Cedrus atlantica dans le traitement des lixiviats. Pour cette raison, nous nous sommes donc intéressés aux sciures du bois de Moringa oleifera compte tenu des propriétés coagulantes et floculantes de ses graines comme l'a stipulé Fatombi et al.,2007. L'objectif de ce travail consiste à proposer un essai de traitement des lixiviats du lieu d'enfouissement sanitaire de Ouèssè/Ouidah (Sud Bénin) à partir de trois types de coagulants, issus d'un même biomatériau naturel : la sciure du bois de Moringa oleifera.

avons travaillé avec la sciure de bois brute. Elle a été lavée à l'eau distillée, filtrée sur Büchner et séchée à l'étuve à $100^{\circ} \mathrm{C}$ jusqu'à l'obtention d'une poudre dénommée $(\mathrm{SBb})$ (figure $\mathrm{n}^{\circ} 2$ ). Ensuite, nous avons procédé à l'activation chimique de la sciure de bois brute par de la soude: une quantité de $100 \mathrm{~g}$ de (SBb) est dispersée dans $0,5 \mathrm{~L}$ de soude $3 \mathrm{~N}$. Le mélange est maintenu ensuite sous agitation magnétique modéré, à température ambiante $\left(25^{\circ} \mathrm{C}\right)$, pendant 1 heure et laissé reposer durant 24 heures (Tahar Bennama et al, 2010). Ensuite, le dépôt est lavé à l'eau distillée afin d'éliminer toute trace de base et sucre hydrolysé jusqu'à un $\mathrm{pH}$ proche de la neutralité. Après filtration sur Büchner, la sciure ainsi récupérée est séchée à $100^{\circ} \mathrm{C}$ et appelée sciure de bois activée (SBa) (figure ${ }^{\circ} 2$ ). Enfin, la sciure brute a été calcinée à $500^{\circ} \mathrm{C}$ dans un four de marque Nabertherm C290 ; le résidu est appelé sciure de bois calcinée (SBc) (figure $n^{\circ} 2$ ). 


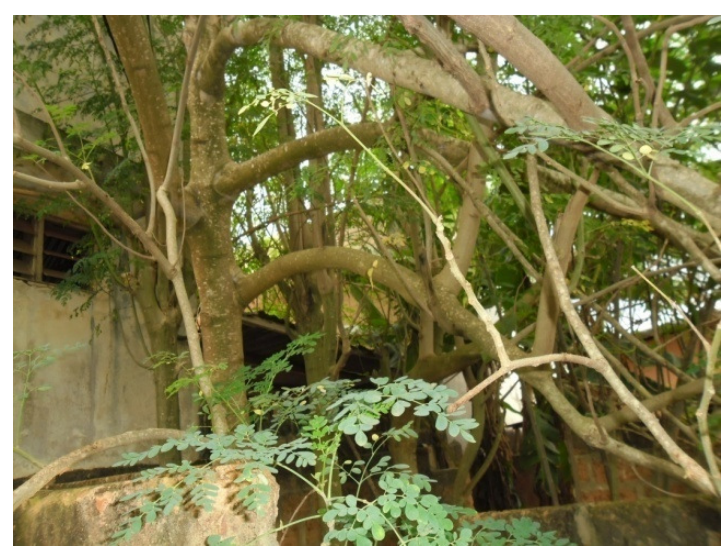

Fiaure 1: Les plantes de Morinaa oleifera

Méthodes d'analyses: La détermination des doses optimales des différents biomatériaux dans les lixiviats a été effectuée par des essais de traitement au jar-test (Folkard et al, 2002; Fatombi et al, 2007). La température (T), le pH, les solides totaux dissous (TDS) et la conductivité ont été mesurés par un multi paramètre de marque WTW 340i ( $\mathrm{pH} /$ Oxymètre). La couleur et la turbidité ont été également mesurées par le colorimètre $\mathrm{HACH}$ DR/890. La Demande Chimique en Oxygène (DCO) dans les échantillons de lixiviat est déterminée à l'aide du spectrophotomètre AL 800 de marque Aqualytic,

\section{RÉSULTATS ET DISCUSSIONS}

Pour déterminer les doses optimales des trois coagulants utilisés, il a été effectué des essais de clarification au jartest (Fatombi et al, 2007). La dose optimale de chaque

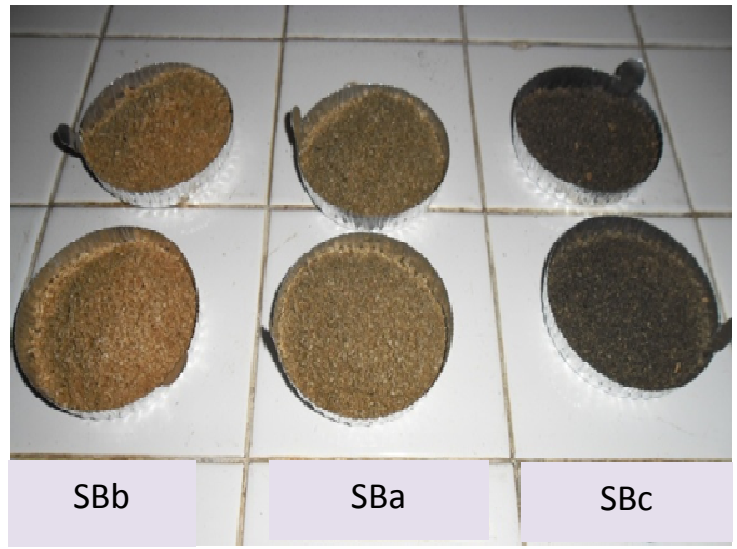

Figure 2 : Aspect visuel des trois types de sciure de bois

méthode par oxydation au dichromate de potassium en milieu $\mathrm{H}_{2} \mathrm{SO}_{4}$ concentré (norme ISO 15705). Le carbone organique total, a été dosé par un COT- mètre de marque Analytic Jena modèle NC $2100 \mathrm{~S}$ par combustion à $800^{\circ} \mathrm{C}$ et dosage du $\mathrm{CO}_{2}$ libéré par Infra-Rouge. L'analyse statistique des données obtenues a été effectuée avec le logiciel Microsoft Office Excel 2007. La variation des paramètres étant faible pendant la période d'étude nous avons utilisé les moyennes obtenues pour la suite de l'étude.

coagulant et pour chaque échantillon de lixiviat correspond au minimum de chacune des courbes obtenues (figures 3,4 ).

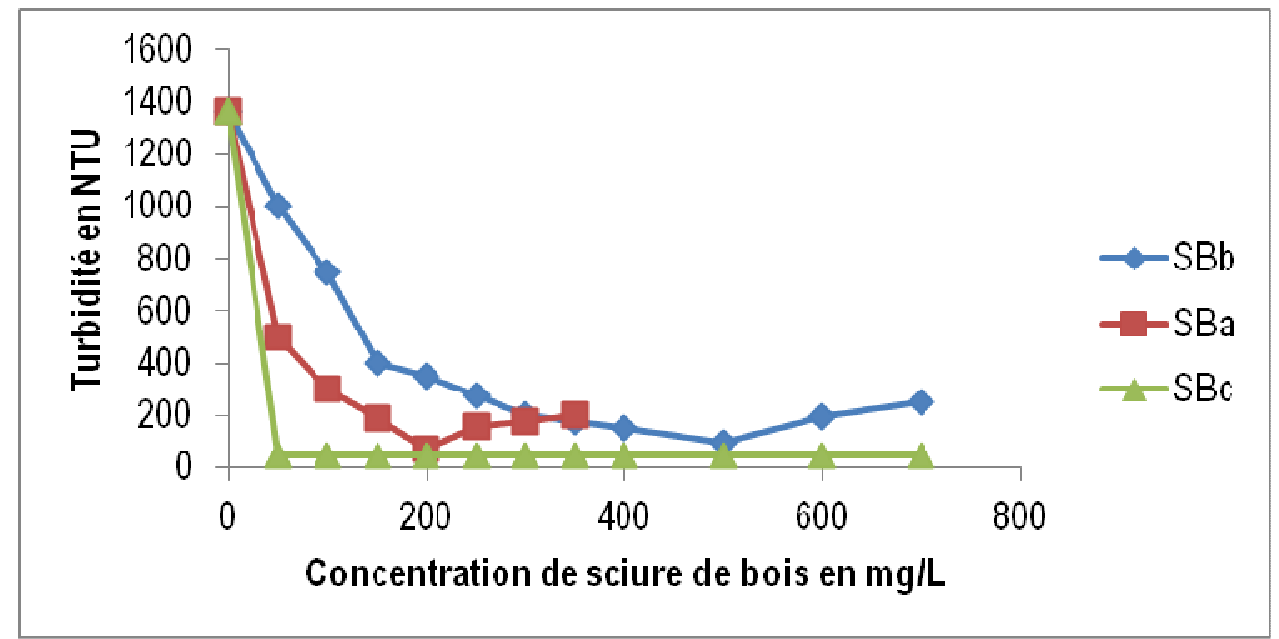

Figure 3 : Variation de la turbidité du lixiviat 1 en fonction des doses de sciure du bois 


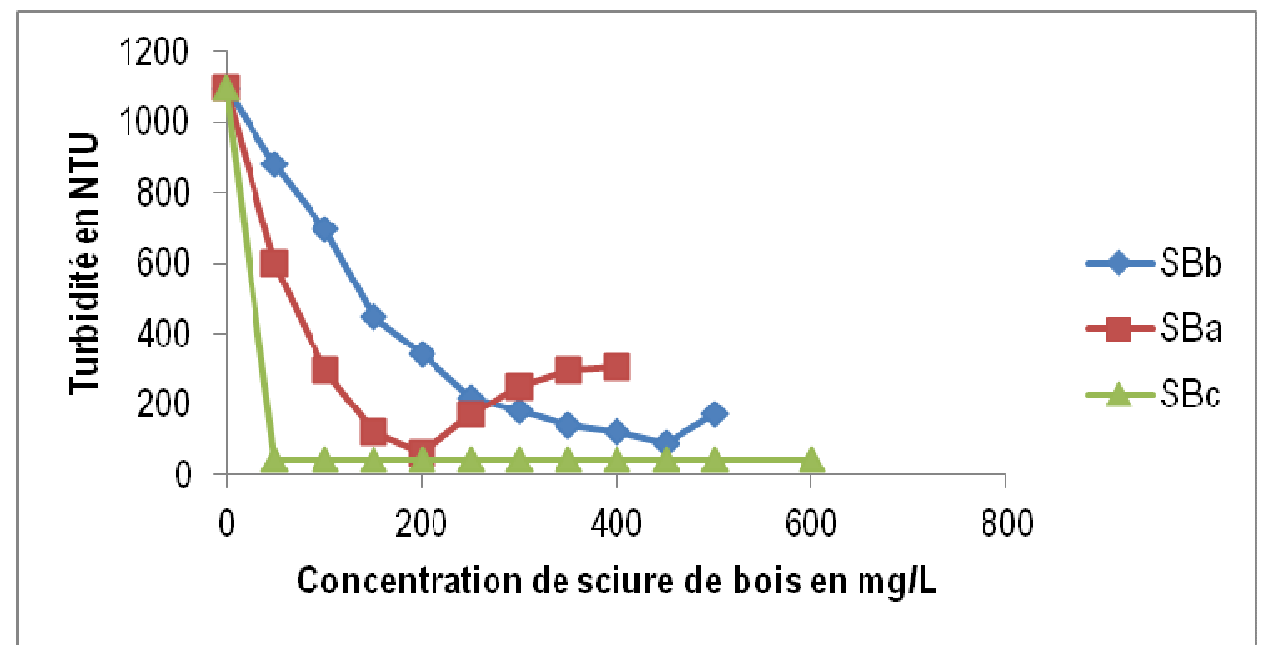

Figure 4 : Variation de la turbidité du lixiviat 2 en fonction des doses de sciure du bois

Les doses optimales de sciure de bois obtenues lors du traitement du lixiviat 1 de turbidité initiale 1360 NTU sont de : $500 \mathrm{mg} / \mathrm{L}$ pour la sciure du bois brute, $200 \mathrm{mg} / \mathrm{L}$ pour la sciure du bois activée et $50 \mathrm{mg} / \mathrm{L}$ pour la sciure du bois calcinée avec des turbidités résiduelles respectives de 95 NTU, 75 NTU et 50 NTU (figure 3). Les doses optimales de sciure de bois obtenues lors du traitement du lixiviat 2 de turbidité initiale 1100 NTU sont de : $450 \mathrm{mg} / \mathrm{L}$ pour la sciure du bois brute, $200 \mathrm{mg} / \mathrm{L}$ pour la sciure du bois activée et $50 \mathrm{mg} / \mathrm{L}$ pour la sciure du bois calcinée avec des turbidités résiduelles respectives de 90 NTU, 65 NTU et 45 NTU (figure 4). Des différents résultats, il ressort que les doses optimales obtenues pour les deux échantillons de lixiviat, dépendent des différents coagulants (sciure de bois brute, sciure de bois activée et sciure de bois calcinée). De la même manière, les turbidités résiduelles obtenues pour les différents échantillons dépendent également des différents coagulants. Ces doses optimales obtenues, ne sont pas liées nécessairement à la turbidité initiale du lixiviat brut. L'augmentation de la turbidité observée après l'obtention de la dose optimale des sciures de bois brute et activée pourrait être due au remplacement d'une turbidité colloïdale par une turbidité particulaire et ce à cause de la présence de la matière organique. La saturation observée après la concentration optimale dans le cas de la sciure du bois calcinée pourrait s'expliquer par le fait que la calcination à $500^{\circ} \mathrm{C}$ élimine les matières organiques dans les sciures. De tout ce qui précède, du point de vue turbidité, nous pouvons dire que la sciure du bois calcinée de Moringa oleifera traite mieux les lixiviats que les sciures du bois brute et celle activée du même biomatériau. Pour la suite des travaux, nous avons mesuré le carbone organique total et la demande chimique en oxygène dans les deux échantillons de lixiviat avant et après le traitement par les différentes sciures de bois. Les résultats obtenus sont représentés par les figures ci-dessous. 


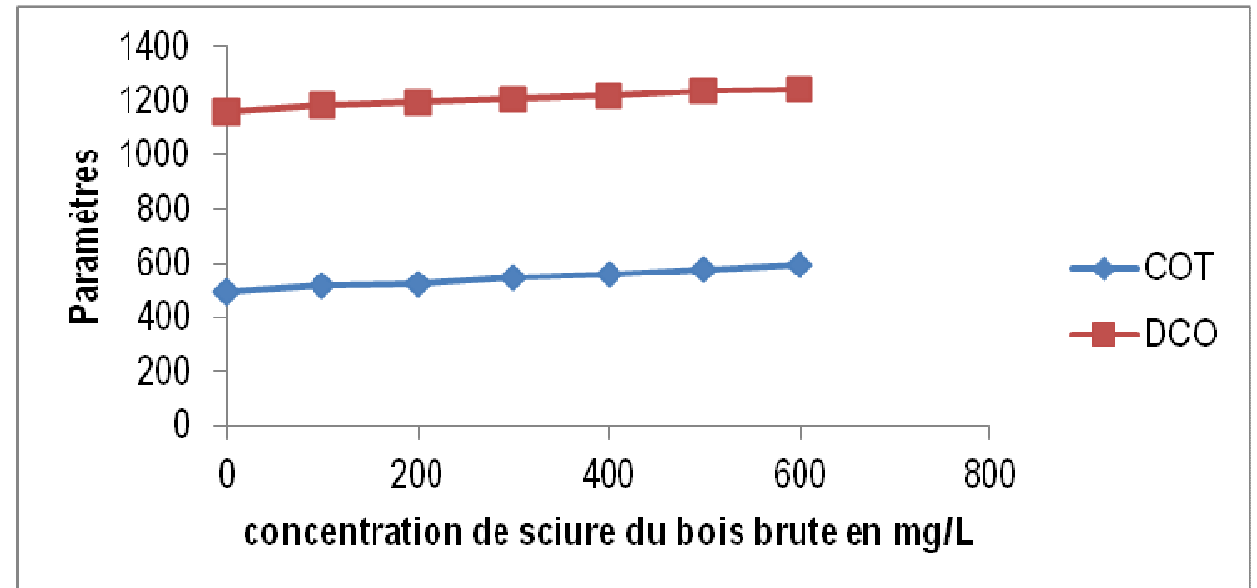

Figure 5 : Variation du COT (mg/L) et de la DCO $\left(\mathrm{mg}^{\prime} \mathrm{L} \mathrm{de} \mathrm{O}_{2}\right)$ du lixiviat 1 en fonction de la dose de sciure de bois brute.

La figure 5 montre que les valeurs du Carbone Organique Total et de la Demande Chimique en Oxygène dans le lixiviat 1 ont connu une légère augmentation lors de l'introduction des différentes doses de sciure de bois brute.

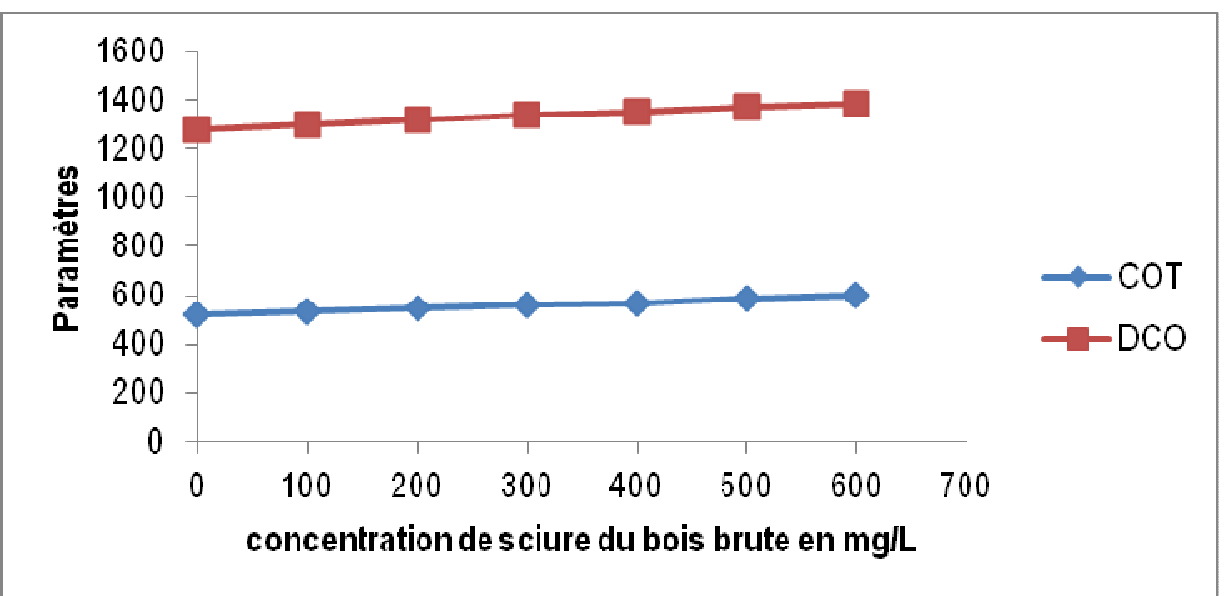

Figure 6 : Variation du COT $(\mathrm{mg} / \mathrm{L})$ et de la DCO $\left(\mathrm{mg} / \mathrm{L}\right.$ de $\left.\mathrm{O}_{2}\right)$ du lixiviat 2 en fonction de la dose de sciure de bois brute.

La figure 6 montre que les valeurs du Carbone Organique Total et de la Demande Chimique en Oxygène dans le lixiviat 2 ont connu une légère augmentation lors de l'introduction des différentes doses de sciure de bois brute. 


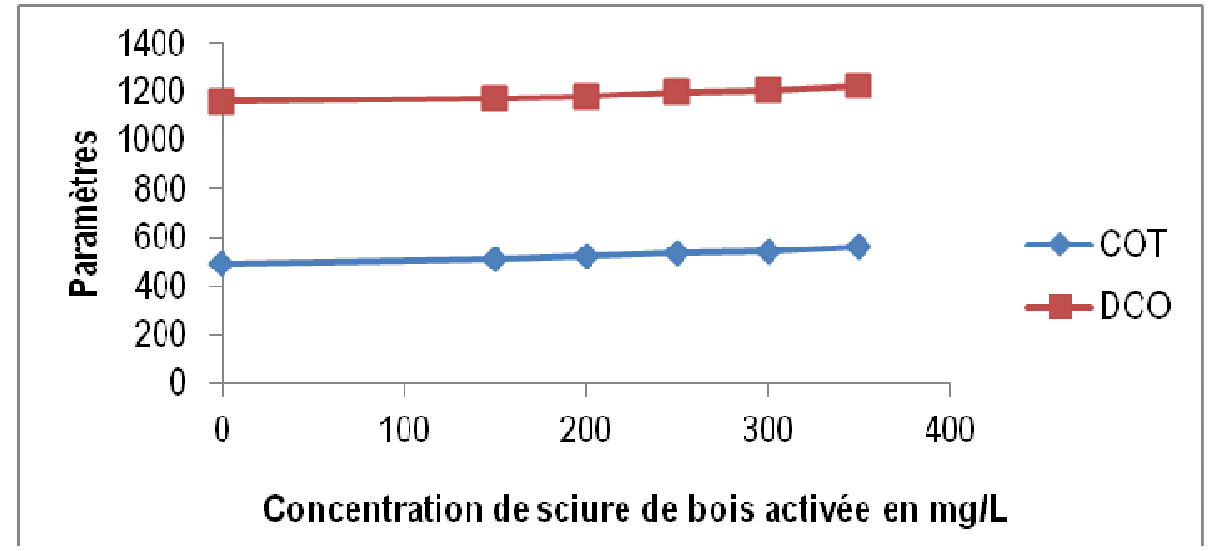

Figure 7 : Variation du COT $(\mathrm{mg} / \mathrm{L})$ et de la DCO $\left(\mathrm{mg} / \mathrm{L}\right.$ de $\left.\mathrm{O}_{2}\right)$ du lixiviat 1 en fonction de la dose de sciure de bois activée

La figure 7 montre que les valeurs du Carbone Organique Total et de la Demande Chimique en Oxygène dans le lixiviat 1 ont connu une légère augmentation lors de l'introduction des différentes doses de sciure de bois activée.

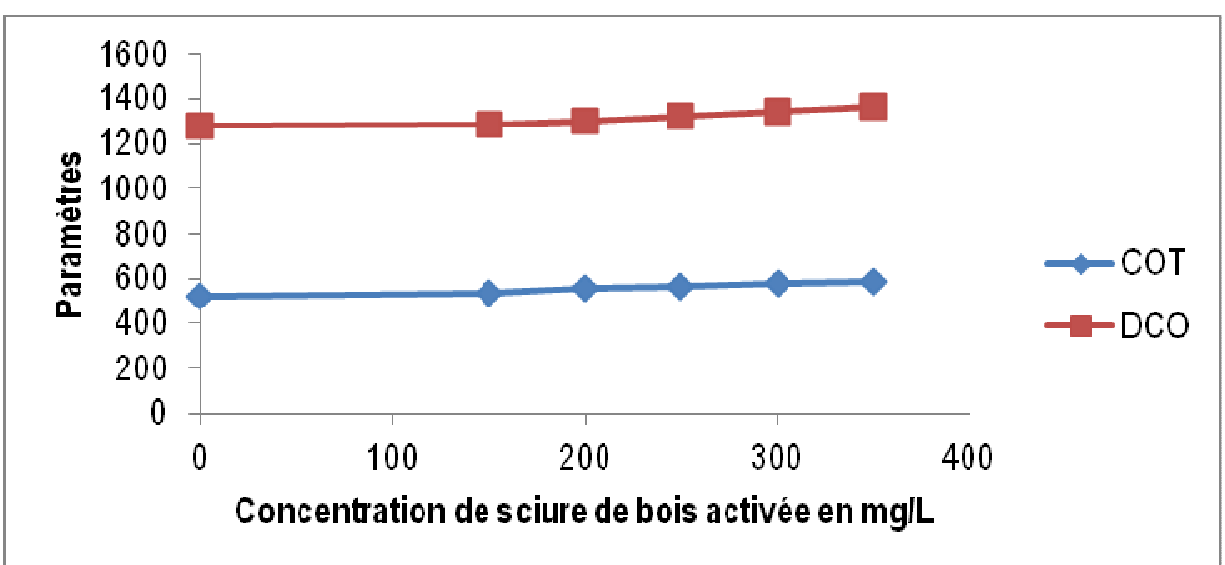

Figure 8 : Variation du COT $(\mathrm{mg} / \mathrm{L})$ et de la DCO $\left(\mathrm{mg} / \mathrm{L}\right.$ de $\left.\mathrm{O}_{2}\right)$ du lixiviat 2 en fonction de la dose de sciure de bois activée

La figure 8 montre que les valeurs du Carbone Organique Total et de la Demande Chimique en Oxygène dans le lixiviat 2 ont connu une légère augmentation lors de l'introduction des différentes doses de sciure de bois activée. 


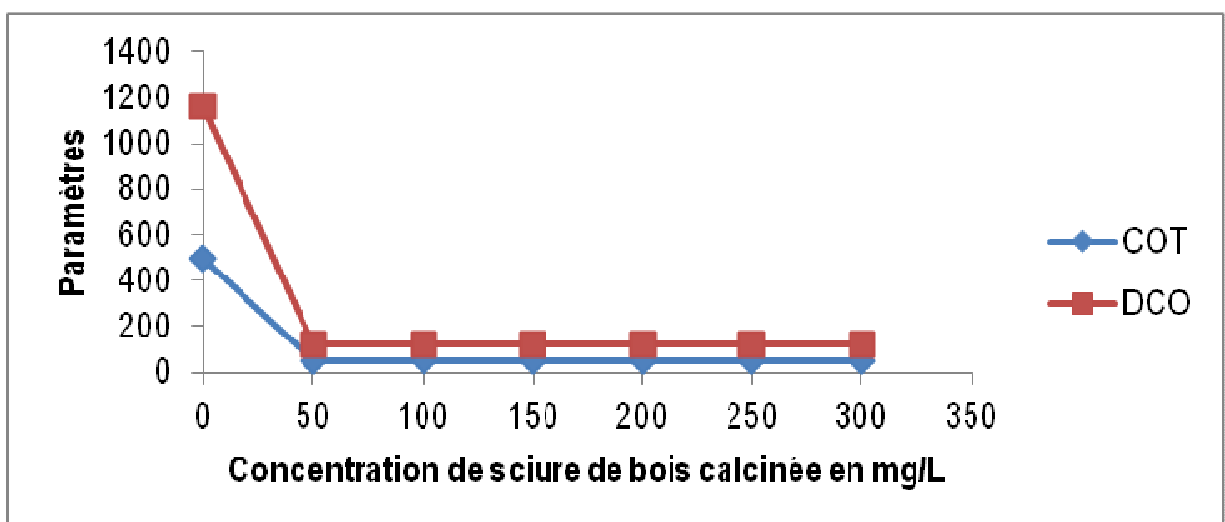

Figure 9 : Variation du COT $(\mathrm{mg} / \mathrm{L})$ et de la DCO $\left(\mathrm{mg} / \mathrm{L}\right.$ de $\left.\mathrm{O}_{2}\right)$ du lixiviat 1 en fonction de la dose de sciure de bois calcinée

La figure 9 montre que les valeurs du Carbone Organique Total et de la Demande Chimique en Oxygène dans le lixiviat 1 ont connu une diminution considérable lors de l'introduction des différentes doses de sciure de bois calcinée.

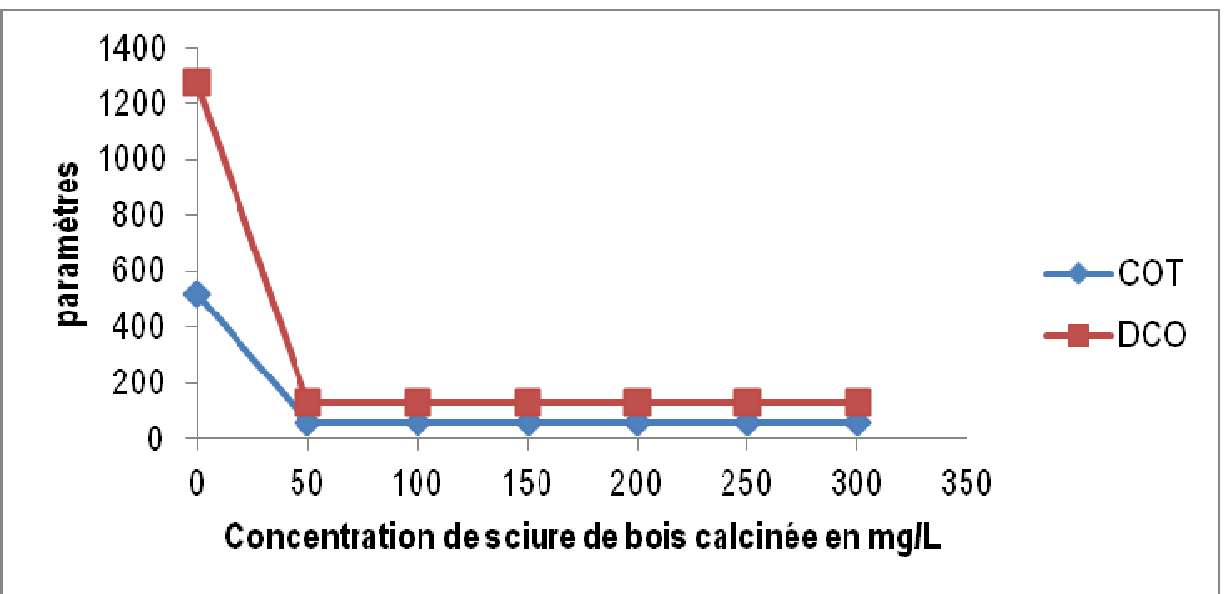

Figure 10 : Variation du COT (mg/L) et de la DCO $\left(\mathrm{mg} / \mathrm{L} \mathrm{de} \mathrm{O}_{2}\right)$ du lixiviat 2 en fonction de la dose de sciure de bois calcinée.

La figure 10 montre que les valeurs du Carbone Organique Total et de la Demande Chimique en Oxygène dans le lixiviat 2 ont connu une diminution considérable lors de l'introduction des différentes doses de sciure de bois calcinée. Une analyse minutieuse des résultats des figures 5, 6, 7 et 8 montre que les sciures du bois brute et activée, diminue la valeur de la turbidité dans les lixiviats, mais augmente leur teneur en matières organiques. Ceci confirme l'hypothèse selon laquelle les valeurs des turbidités augmentent après un minimum pour ces coagulants. Ces résultats confirment ceux de Fatombi et al, (2007) qui ont révélé qu'après traitement, les eaux obtenues sont plus riches en matières organiques. Des figures 9 et 10, nous pouvons déduire que, les surnageants issus des lixiviats traités par la sciure de bois calcinée ont une faible quantité de matières organiques. De tout ce qui précède, nous pouvons dire que la sciure du bois calcinée de Moringa oleifera baisse considérablement les valeurs des matières organiques dans les lixiviats et est donc le coagulant approprié pour clarifier les lixiviats alors que les sciures du bois brute et celle activée du même matériau augmentent légèrement ces matières organiques dans ces lixiviats. Le taux d'élimination de la matière organique obtenu à l'aide des sciures de bois calcinées $(90 \%)$ est supérieur à ceux obtenus dans la littérature: (54\% et $30 \%$ respectivement sous condition aérobie et sous condition anaérobie par Hakkou et al, (2001 a ; 2001 b) ; 60 \% obtenu par Henry \& Prasad (2000) et 84,36 \% par Tahar Bennama et al, (2010). La matière organique est un paramètre qui 

physico-chimique des lixiviats du lieu d'enfouissement sanitaire (LES) de Ouèssèl Ouidah (Sud Bénin)

permet d'estimer la biodégradabilité des différents lixiviats. Elle représente l'utilisation effective des microorganismes et traduit l'expression mesurable du potentiel d'interactions (Toklo et al, 2015). Au sens strict, la biodégradabilité englobe deux processus : d'une part l'utilisation microbienne directe ou après décomposition de composés pour la biosynthèse de nouveau matériel cellulaire, et d'autre part la minéralisation pour obtenir de l'énergie et des nutriments inorganiques. Une diminution de cette réactivité est essentielle pour ralentir la persistance de la matière organique dans un milieu. Nous avons également étudié l'influence du traitement des lixiviats par la sciure du bois calcinée sur la température et le $\mathrm{pH}$. Les différents résultats ont été reportés sur les figures 11 et 12 .

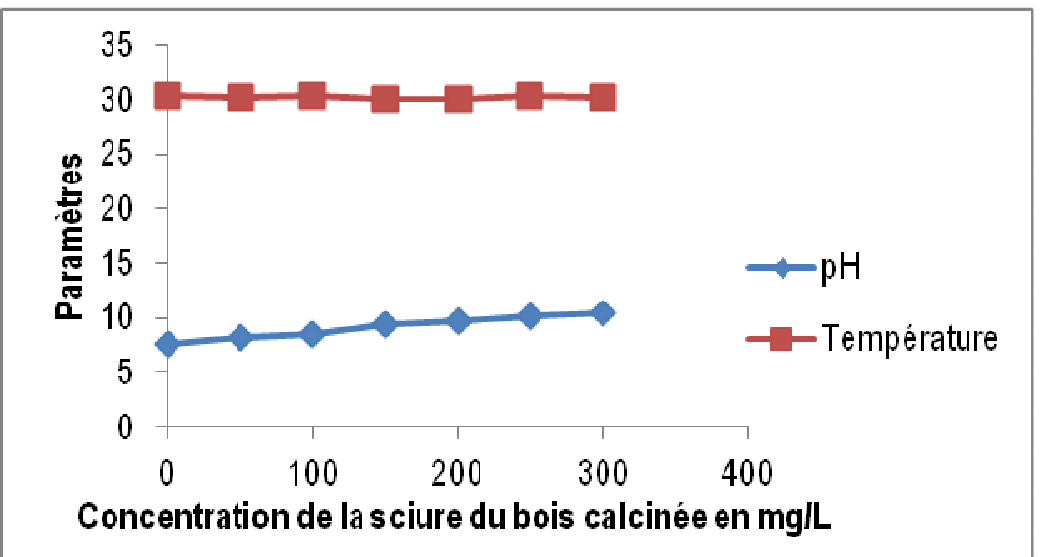

Figure 11 : Variation de la température $\left({ }^{\circ} \mathrm{C}\right)$ et du pH du lixiviat 1 en fonction de la dose de la sciure de bois calcinée.

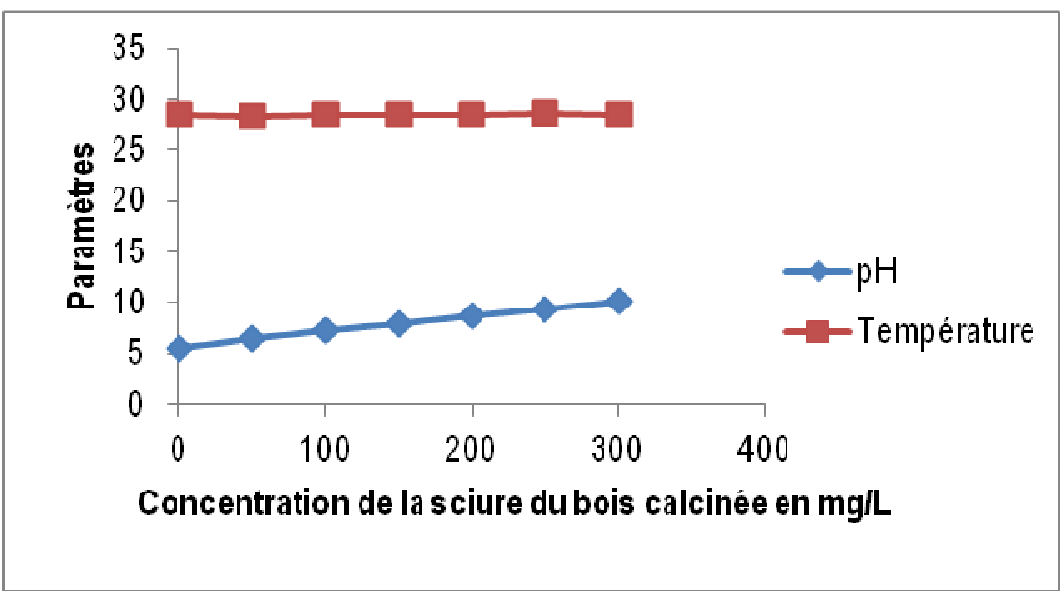

Figure 12 : Variation de la température $\left({ }^{\circ} \mathrm{C}\right)$ et du pH du lixiviat 2 en fonction de la dose de sciure de bois calcinée.

Des figures 11 et 12, on peut déduire que le traitement des lixiviats par les sciures de bois calcinées n'influence pas la température mais influence le $\mathrm{pH}$ des lixiviats traités. Nous remarquons sur les deux figures que le $\mathrm{pHa}$ augmenté jusqu'à atteindre une valeur basique. Cette basicité pourrait s'expliquer par le fait que la sciure du bois calcinée apporte aux lixiviats traités des aluminosilicates alcalins (Chinna et al, 2015), correspondant à la libération des ions de nature basique
(Dusart et al, 1991 ; Faby et al, 1993 ; Piplode et al, 2015) dans les lixiviats. Après l'identification du coagulant (le biomatériau qui traite mieux les lixiviats) et la détermination de sa concentration optimale, nous avons effectué la mesure de la matière en suspension (MES), des solides totaux dissous (TDS), de la couleur et de la conductivité électrique. Les résultats obtenus sont consignés dans le tableau suivant. 
Josse et al. J. Appl. Biosci. 2015 Valorisation des sciures du bois de Moringa oleifera dans le traitement physico-chimique des lixiviats du lieu d'enfouissement sanitaire (LES) de Ouèssèl Ouidah (Sud Bénin)

Tableau 1 : Teneur de quelques paramètres avant et après traitement par les sciures de bois calcinées

\begin{tabular}{|l|l|l|l|}
\hline Paramètres & Avant traitement & Après traitement & Echantillons \\
\hline MES en $\mathrm{mg} / \mathrm{L}$ & $470,0 \pm 3,0$ & $180,0 \pm 2,8$ & \\
\hline TDS en $\mathrm{mg} / \mathrm{L}$ & $7024,0 \pm 3,4$ & $805,2 \pm 2,4$ & \\
\hline Couleur en Pt Co & $6760 \pm 10$ & $2045 \pm 8$ & \multirow{2}{*}{ Lixiviat 1} \\
\hline Conductivité en $\mathrm{mS} / \mathrm{cm}$ & $14,05 \pm 0,12$ & $16,35 \pm 1,15$ & \\
& & & \multirow{2}{*}{ Lixiviat 2 } \\
\cline { 1 - 2 } MES en $\mathrm{mg} / \mathrm{L}$ & $528,0 \pm 3,7$ & $204,0 \pm 1,6$ & \\
\hline TDS en $\mathrm{mg} / \mathrm{L}$ & $11914,00 \pm 7,11$ & $1346,0 \pm 4,0$ & \\
\hline Couleur en Pt Co & $8736 \pm 12$ & $2685 \pm 7$ & \\
\hline Conductivité $\mathrm{mS} / \mathrm{cm}$ & $218,00 \pm 2,07$ & $221,65 \pm 1,95$ & \\
& & & \\
\hline
\end{tabular}

Les résultats obtenus après traitement avec la dose optimale de la sciure de bois calcinée, nous amène à dire que le coagulant a baissé la MES, le TDS et la couleur avec une diminution respective de $61,70 \%, 88,53 \%$ et $69,74 \%$ et a augmenté la conductivité électrique des différents échantillons. Cette augmentation de la

\section{CONCLUSION}

Cette étude montre que les coagulants tels que les sciures de bois brute, activée et calcinée de Moringa oleifera peuvent être utilisés comme support de traitement des lixiviats. Les résultats obtenus ont montré que la sciure de bois calcinée permet d'obtenir une meilleure clarification des lixiviats. Après le traitement, les sciures de bois brute et activée ont diminué la turbidité et augmenté la matière organique. Ils sont donc peu efficaces dans la clarification du traitement des lixiviats. Les sciures de bois calcinées permettent une diminution

\section{RÉFÉRENCES}

Ajmal M., Rao R.A.K., Siddiqui B.A. 1996, Studies on removal and recovery of $\mathrm{Cr}(\mathrm{VI})$ from electroplating wastes Wat. Res, 30(6) 1 4781482. 48.

Ajmal M., Khan A.H., Ahmad S., Ahmad A. 1998, Role of sawdust in the removal of copper(II) from industrial wastes. Wat. Res, 32(10) 3085-3091. 49.

Baun A., Ledin A., Reitzel L.A., Bjerg P.L., Christensen T. H., 2004. Xenobiotic organic compounds in leachates from ten Danish MSW landfillschemical analysis and toxicity tests. Water Res, (38): 3845-3858.

Chatterjee. S., Asthana R. K., Tripathi A. K., Singh S.P. 1996, Metal removal by selected sorbents Biochem, 31 (5) 457- 462. 51.

Chinna R.P. and Gundala P.H., 2015 Analysis of Water Quality Parameters of Lakshmipuram tank, conductivité est due au fait qu'au cours du processus de traitement, il y a eu minéralisation du lixiviat par les sciures calcinées. Ceci confirme l'hypothèse selon laquelle le $\mathrm{pH}$ a augmenté après le traitement. La sciure du bois de Moringa oleifera constitue donc le coagulant approprié pour un meilleur traitement des lixiviats.

considérable des matières organiques $(90 \%)$, de la MES $(61,70 \%)$, du TDS $(88,53 \%)$, de la couleur $(69,74 \%)$ et de la turbidité (95\%). Cette diminution pourrait être due au fait que la calcination à $500^{\circ} \mathrm{C}$, élimine les matières organiques dans les sciures et facilite la coagulation. L'avantage de cette étude, est que le traitement des lixiviats à la sciure du bois calcinée de Moringa oleifera n'exige pas une grande quantité de coagulant et n'introduit pas de matière organique dans les lixiviats après le traitement.

Anantapuramu, Andhra Pradesh, India, J. Chem. Sci., 5(4), 26-32.

Dusart O., Mazet M., Souabi S., Yacoubi A., 1991 Adsorption of aminoacids on activated carbon in water: equilibrium parameters determination by different equations Tribune de l'eau, 3 (42) (539) 29-34.

Faby J. A. ; Eleli A., 1993 Utilisation de la graine de Moringa: essai de floculation en laboratoire et en vraie grandeur C.I.E.H, 1-99.

Folkard, G., Sutherland J. et Al-Khalili R. S., 2002. La clarification de l'eau par coagulation en utilisant les graines du Moringa oleifera. In : L. Fugie, 2002. L'arbre de la vie, les multiples usages du Moringa. CWS/CTA. Dakar, Sénégal, pp 79-82.

Hakkou R., Wahbi M., Bachnou A., Elamari K., Hanich L. \& Hibti M., 2001. Impact de la décharge publique de Marrakech (Maroc) sur les 
ressources en eau. Bulletin of Engineering Geology and Environment, 60: 325 - 336

Holan Z.R., Volesky B. 1995, Accumulation of cadmium, lead, and nickel by fungal and wood biosorbents Applied Biochem. Biotechnol, $53:$ 133-146. 50.

Fatombi J.K., Josse R.G., Wotto V., Aminou T., Coulomb B., 2007. Paramètres physico-chimiques de l'eau d'okpara traitée par les graines de Moringa oleifera. J. Soc. Ouest-Afr. Chim 023; 75-79.

Fatombi J.K., Josse R.G., Yehouenou B., Wotto V., Aminou T., 2007. Traitement des eaux de surface par les coagulants à base de coco ; J. Soc. Ouest-Afr. Chim $023 ; 81-87$.

Piplode S. and Singh V.B., 2015. Physico-chemical Evaluation of Narmada River Water at Khalghat MP, India, Res. J. Chem. Sci., 5(5), 24-26

TOKLO R.M., JOSSE R.G., TOPANOU N., TOGBE A.F.C., DOSSOU-YOVO P. and COULOMB B., 2015. Physico-chemical characterization of the leachates of a discharge: case of sanitary burying place of Ouèssè-Ouidah (South of
Benin). International Journal of Innovation and Applied Studies, Vol. 13 N$^{\circ} 4$, PP: 921-928.

Bennama T., Younsi A., Derriche Z. et Debab A., 2010. Caractérisation et traitement physico-chimique des lixiviats de la décharge publique d'El Kerma (Algérie) par adsorption en discontinu sur de la sciure de bois naturelle et activée chimiquement. Water Qual. Res. J. Can. Volume 45, No. 1, 81-90

Toklo R. M., Josse R. G., Dossou-Yovo P., Topanou N., Yehouenou B. and Akogbeto P., 2015. Assessment of the Degree of Surface and Ground water pollution in the Landfill area of Ouèssè-Ouidah South of Benin. Res. J. chem. .sci., 5(9),34-41

Zalaghi A., F. Lamchouri, H. Toufik, M. Merzouki, 2014. Valorisation des matériaux naturels poreux dans le traitement des Lixiviats de la décharge publique non contrôlée de la ville de Taza. J. Mater. Environ. Sci. 5 (5) 1643-1652 